\title{
We have it
}

\section{History has been made with the discovery of a Higgs-like particle at CERN.}

Physics is not usually theatre. But the drama of the seminar held at CERN on 4 July 2012, whether watched via webcast or in the CERN auditorium itself, was outstanding. Both generalpurpose detectors, ATLAS and CMS, had something to say, and speculation had been rife in the days ahead of the announcement.

Following the hints that had emerged in CERN data at the end of last year, Director General Rolf-Dieter Heuer had confidently stated that there would be a definitive resolution by the end of 2012 as to whether these hints were the signal of a newly discovered particle or not. Fortunately the superb performance of the Large Hadron Collider - CERN's $27-\mathrm{km}$ accelerator, smashing protons at a centre-of-mass energy of $8 \mathrm{TeV}$ - has continued this year, and a significant body of data, collected right up to mid-June, was added into the experiments' analyses before the July presentation. There was good reason to be optimistic: perhaps that gentle hint of something had become somewhat stronger, and might be on course, by the end of the year, to become an indisputable signal.

Astoundingly, though, on 4 July the signal was already there. One after the other, collaboration spokesmen Joe Incandela and Fabiola Gianotti presented the data from CMS and ATLAS, respectively, both datasets indicating the existence of a particle at a mass around $126 \mathrm{GeV}$. As Incandela's powerpoint slides flicked on to the invariant-mass distribution of pairs of photons picked up in the CMS detector, the audience in

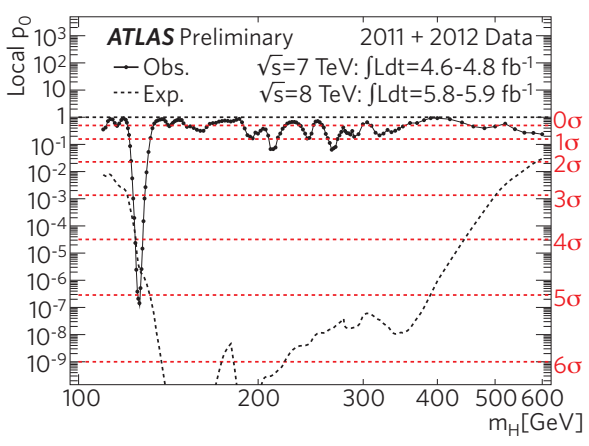

Take the plunge: for data presented by the ATLAS collaboration, the probability of background to produce a signal-like excess (solid line), for all Higgs-boson masses $\left(m_{H}\right)$ tested, is only a few per cent at almost all masses - except for a mass of $126.5 \mathrm{GeV}$, where the probability crashes to 0.0000003 , passing the all-important five-sigma mark for a discovery. Image: Atlas Experiment (C) 2012 CERN

front of him (and around the world, no doubt) gasped. There, around the $126-\mathrm{GeV}$ mark, was the neatest of bumps - the signature of a particle, created in the LHC collisions, decaying into pairs of photons.

The signature pops up in other decay channels too, and overall the significance of the CMS signal checks in at 4.9 sigma. A similar presentation from Gianotti (including the striking probability plot, pictured) reinforced the excitement, with ATLAS demonstrating a 5.0-sigma significance. Rarely do seminar speakers have to pause at regular intervals to allow the applause to subside, but Incandela and Gianotti must have loved every minute of it.

Among that appreciative audience at CERN were a handful of theorists who, nearly fifty years ago, had devised a mechanism of electroweak symmetrybreaking that implies the existence of another boson in the standard model: Peter Higgs, François Englert, Gerald Guralnik and Carl Hagen (although without Tom Kibble, and without Robert Brout who died in 2011). Mobbed by the press after the seminar, Higgs was coy but obviously moved by what might be the vindication of the mechanism that bears his name.

That 'might' is important. CERN physicists are talking of the discovery of a "Higgs-like" particle - because, as yet, although there is something there, it isn't proven what that something is. In fact, it doesn't quite line up with the expectations for a standard-model Higgs boson, or for a Higgs boson built into an extended theory such as supersymmetry. (The LHC has yet to turn up any evidence in favour of supersymmetry, which has ramifications for dark-matter searches, as discussed in a News and Views article in this issue, page 584.) But more data are needed for a proper characterization of the particle (its spin, decay channels and rates, and so on), to understand exactly what it is - or perhaps to signal that we must go back to the drawing board to account for this new particle.

But for the moment, it does seem like the momentous culmination of a fifty-year search. As Heuer concluded on 4 July: "I think we have it".

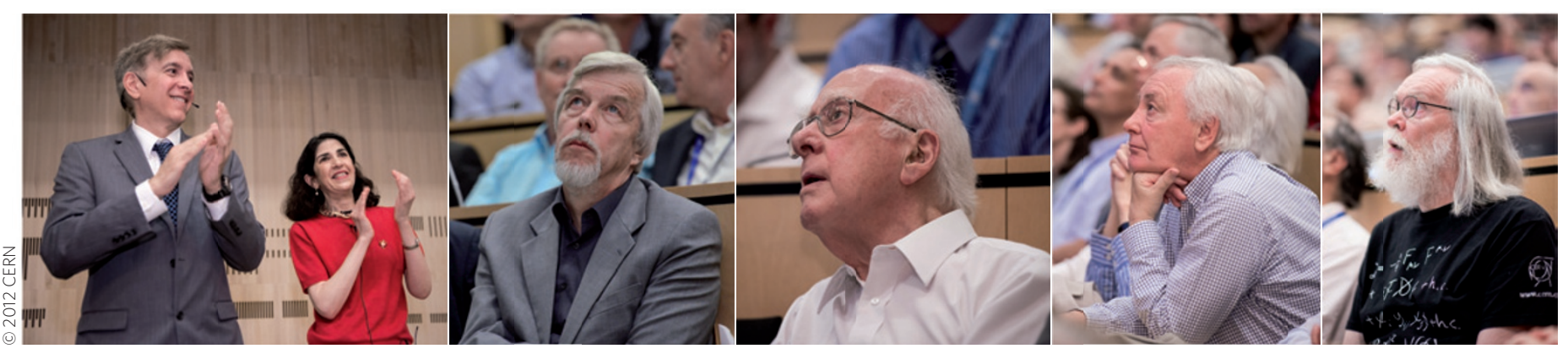

Big day: left to right, CMS and ATLAS spokesmen Joe Incandela and Fabiola Gianotti; CERN Director-General Rolf-Dieter Heuer; Peter Higgs; former LHC project leader Lyn Evans; and theorist John Ellis. 